\title{
Olena Byelozyorova
}

ORCID: 0000-0002-6668-5685

Charkiwer Nationale W.N. Karasin-Universität, Charkiw

https://doi.org/10.19195/0435-5865.146.4

\section{Kommunikative Strategien zur Bewältigung von verbalen Tabus in ZeitzeugInnen-Interviews mit Verfolgten des Nationalsozialismus*}

\begin{abstract}
s
Der vorliegende Beitrag widmet sich den verbalen Tabus sowie den kommunikativen Strategien derer Bewältigung in den lebensgeschichtlichen Interviews mit den NS-ZeitzeugInnen. Die verbalen Tabus werden als Konzepte definiert, die keine explizite Verbalisierung im Diskurs dulden. Die Forschung hat ergeben, dass es grundsätzlich um zwei Tabu-Cluster geht. Zum einen handelt es sich um die traumatische Erfahrung der Verfolgung, die durch die kommunikative Strategie des Verschweigens und die der Euphemisierung bewältigt wird. Zum anderen geht es um den expliziten Ausdruck von negativen Emotionen - solche Tabus werden durch die kommunikative Strategie des Gesichtswahrens aufgefangen. Diese Strategien werden besonders effizient durch den Sprechakt der Andeutung realisiert, der ein diskursiv-sprachliches Zusammenwirken von Sprecher/in und Hörer/ in zur Konstruktion der intendierten impliziten Proposition darstellt.
\end{abstract}

Schlüsselwörter: Andeutung, impliziter Sprechakt, kognitive Operation, kommunikative Strategie, NS-Verfolgung, Tabu

\section{Strategies for managing verbal taboos in contemporary witness interviews with victims of National Socialist persecution}

The article focuses on verbal taboos and communication strategies for their management in autobiographical interviews with victims of National Socialist persecution. Verbal taboos are interpreted here as concepts that exclude their explicit verbalization. In the course of study, it was possible to

* Anhand der Audio- und Video-Interviews aus dem digitalen Interview-Archiv, www.weitererzählen.at und der gekürzten Versionen derselben Interviews auf Lernplattform, https://www.ueber-leben.at/home (Zugriff am 1.07.2020). 
identify two main groups of verbal taboos actualized in this type of discourse. The first group relates to the traumatic experience of the respondents' persecution. This suggests that taboos are managed through implementing silence and euphemization strategies. The second group refers to the verbalization of negative emotions caused by traumatic experiences. For this group of taboos, a face-saving strategy stands for the most relevant one. From the above analysis, a key finding emerged: an effective tool for implementing both strategies is the speech act of hinting - a discursive speech interaction between the addresser and the addressee, whereby they construct an intended implicit proposition.

Keywords: Hint, implicit speech act, cognitive operation, communicative strategy, National Socialist persecution, taboo

Olena Byelozyorova, Lehrstuhl für Deutsch und Französisch, Raum 8-29, Fakultät für Fremdsprachen, Charkiwer Nationale W.N. Karasin-Universität, Maidan Swobody 4, 61022 Charkiw, Ukraine, E-Mail: byelozyorova@yahoo.com.

Received: 30.09.2020, accepted: 8.03.2021

Nathan Fischmann

Wo ist er im Verzeichnis der österreichischen Opfer? [...]

Ich muß mir nicht Dachau, nicht Mühldorf anschauen

Ich muß sein Gesicht finden sein Gesicht anschauen

Konstantin Kaiser ${ }^{1}$

heldentaten einen Vater zu haben (gehabt zu haben) der einen Krieg mitgemacht hat und - schweigsam geworden nichts darüber erzählt hat ist vielleicht besser als einen Vater zu haben

(gehabt zu haben) der einen Krieg mitgemacht hat und gern darüber geredet und gern

von seinen Heldentaten erzählt hat

Elfriede Haslehner ${ }^{2}$

\footnotetext{
1 Kaiser 2017.

${ }^{2}$ Haslehner 2001.
} 


\section{Einleitung}

Die wissenschaftliche Beschäftigung mit dem Thema „Tabu und Geschichte“ hat inzwischen eine lange Tradition und wirft Licht auf viele Facetten dieses Themenkomplexes, ${ }^{3}$ auch im österreichischen Kontext der Bewältigung der NS-Vergangenheit: „In der Auseinandersetzung mit der Geschichte des Nationalsozialismus und Faschismus oder bei den wunden Punkten der Kommunistischen Parteien verweist der Zusammenhang von Tabu und Geschichte auf die Ausblendung von Vorgängen oder Tatsachen aus dem Diskurs, um eine komplexe Realität einfach bewältigen zu können“. ${ }^{4}$ Primär steht aber die Tabuisierung der NS-Vergangenheit durch die „TäterInnen“ bzw. die „MittäterInnen“ im Vordergrund der themenbezogenen Studien, in denen Tabus als „Axiome“ verstanden werden, die „potentiell Gefährliches zudecken“, aber vor allem „potentiell Schmerzhaftes zudecken sollten". ${ }^{5}$ Dabei wird übersehen, dass eine solche Tabudefinition genauso relevant auch für die Erforschung des Schweigens der Opfer wäre, die ihre Traumata der NS-Verfolgung eine lange Zeit verschwiegen haben: „Ich habe nicht darüber gesprochen. Ich wollte auch nicht darüber sprechen, besonders. Ich habe das verschwiegen. Jahrzehntelang. Aber im Kopf war es immer da. Und die nächtlichen Alpträume waren von Zeit zu Zeit auch da". ${ }^{6}$ Die ZeitzeugInnen-Interviews, in denen die „Opfer“ ihre Lebensgeschichten erzählen, sind als Versuch der Enttabuisierung zu verstehen. Dabei wird ersichtlich, dass die Kommunikation zwischen den GesprächspartnerInnen in solchen Interviews keinesfalls soweit reicht, völlig tabufrei zu sein. Andeutungen, euphemistische Ausdrucksweisen, aposiopesische Konstruktionen und absichtlicher Themenwechsel sind Zeichen für das Existieren von verbalen Tabus, die die GesprächspartnerInnen dazu bringen, sich einer verschleiernden Ausdrucksweise zu bedienen. Dieses kommunikative Phänomen weckt Interesse auch aus sprachwissenschaftlicher Sicht.

\section{Zielsetzung, Forschungsmaterial und Methoden}

Der vorliegende Beitrag widmet sich den verbalen Tabus, die in der Kommunikation über die NS-Verfolgung mit den Verfolgten aktualisiert werden, mit dem Ziel festzustellen, welche kommunikativen Strategien zur sprachlichen Bewältigung solcher Tabus von SprecherInnen eingesetzt werden. Unter diesem Blickwinkel wurden sämtliche deutschsprachigen ZeitzeugInnen-Interviews des digitalen

3 Vgl. Bettelheim / Streibel 1994; Meier 2010; Pelinka / Weinzierl 1987; Ziegler / KannonierFinster 1993.

${ }^{4}$ Bettelmann / Streibel 1994: 10-11.

5 Pelinka 1994: 21.

${ }^{6}$ Interview mit Herbert Traube (2019): 51:25, https://www.weitererzaehlen.at/interviews/ herbert-traube (Zugriff am 1.07.2020). 
Erinnerungsprojektes „weiter_erzählen“ analysiert (insgesamt 149, 7 davon auBerdem in gekürzter Form auf der Lernplattform www.ueber-leben.at ${ }^{7}$ ), das vom österreichischen Bundesministerium für Bildung, Wissenschaft und Forschung (BMBWF) 2019 anlässlich des Internationalen Holocaust-Gedenktages vorgestellt wurde. Es beinhaltet insgesamt 160 aufgezeichnete Erinnerungen von im Nationalsozialismus verfolgten Menschen, die einen Bezug zu Österreich haben. ${ }^{8}$

Unter verbalen Tabus werden hier Konzepte verstanden, die keine explizite Verbalisierung im Diskurs dulden. Angemessene Instrumente zur Analyse dieses Phänomens bietet die kognitive Sprachpragmatik, und zwar ihre Erkenntnisse zu impliziten Sprechakten ${ }^{9}$ und zu den kognitiven Operationen, ${ }^{10}$ die in den letzteren im Spiel sind. Implizite Sprechakte weisen das Vorhandensein von mehreren Propositionen auf - einer expliziten und mindestens einer impliziten. Aus der expliziten Proposition schlussfolgert der Hörer/die Hörerin die vom Sprecher intendierte/-n impliziten Propositionen (in der relevanten Forschungsliteratur auch Implikaturen genannt ${ }^{11}$ ). Implizites Sprechen ermöglicht die Kommunikation über tabuisierte Sachverhalte.

Ein höchst effizienter Sonderfall einer solchen indirekten tabubezogenen Kommunikation ist die Andeutung, ein diskursiv-sprachliches Zusammenwirken von Sprecher/in und Hörer/in, wobei sie als gleichberechtigte PartnerInnen am Prozess der Sinnkonstruktion teilnehmen. Die Andeutung ist eine Sprachhandlung, bei der Sprecher/in und Hörer/in ihre Aufmerksamkeit auf die explizite Proposition und Illokution richten und fokussieren, um dann durch das harmonische Zusammenspiel mehrerer kognitiver Operationen (Prominenz, Fokussierung, Spezifizität und Perspektive) die implizite/-n Proposition/-en samt impliziten Illokutionen zu erschließen: ,The speaker's and hearer's action involves the directing and focusing of attention $(\rightarrow)$. In successful communication, they manage to coordinate this action and focus attention on the same conceived entity". ${ }^{12} \mathrm{Im}$ Sprechakt der Andeutung wird auf der Basis seiner expliziten und impliziten Propositionen sowie expliziten und impliziten Illokutionen seine implizite Proposition profiliert (dadurch wird die kognitive Operation der Prominenz vollzogen, die ,imposition ,of ,profile' on a ,base““" darstellt. ${ }^{13}$ Die kognitive Operation der Fokussierung bezieht sich im Sprechakt der Andeutung auf die salienten Argumente der expliziten Proposition sowie die eventuell vorhandenen non-verbalen Indikatoren der Andeutung. Die kognitive Operation der Spezifizierung bezieht sich auf die „Transparenz“ der jeweiligen Andeutung, deren Grad variieren kann. Die kognitive Operation der Perspektive bestimmt die Lage des jeweiligen Referenzpunktes,

\footnotetext{
${ }^{7}$ https://www.ueber-leben.at/home (Zugriff am 1.07.2020).

8 https://www.weitererzaehlen.at (Zugriff am 1.07.2020).

9 Vgl. Liedtke 1995; Rolf 2013; Zimmermann / Müller 1977: 238-254.

10 Langacker 2001: 143-148.

11 Grice 1993: 243-265.

12 Langacker 2001: 144.

13 Langacker 1990: 5.
} 
wodurch zwischen retrospektiven, introspektiven und prospektiven Andeutungen unterschieden werden kann.

\section{Verbale Tabus und kommunikative Strategien zu deren Bewältigung}

Die empirische Analyse der genannten ZeitzeugInnen-Interviews lässt darauf schließen, dass durch Andeutungen hier mehrere verbale Tabus aufgefangen werden. Es wäre grundsätzlich zwischen zwei großen Tabu-Clustern zu unterscheiden.

\subsection{Tabuisierung der traumatischen Erfahrungen der NS-Verfolgung: Strategie des Verschweigens und die Euphemisierungsstrategie}

Der erste Tabu-Cluster bezieht sich auf die eigenen traumatischen Erfahrungen der Verfolgung. Hier lassen sich zwei wichtige kommunikative Strategien beobachten - die des Verschweigens und die der Euphemisierung. Die kommunikative Strategie des Verschweigens lässt vermuten, dass dem/der Interviewten des Öfteren die Worte fehlen, um das Ausmaß des erlebten Leidens zu beschreiben, dass keine Worte sprachgewaltig genug sind, um das wiederzugeben. Darum bedient er/sie sich der Andeutungen mit salienten Argumenten der expliziten Proposition, die z.B. durch die Pronomen es, das, was, nichts, alles ausgedrückt sind, z.B.:

(1) Jehudith Hübner: Aber zwei Dinge habe ich nicht... Ich kann nicht vergessen, was geschehen ist, und werde es nicht vergessen, und man soll es nicht vergessen, und ich sage es auch meinen Kindern, sie sollen es nicht vergessen, denn das war tierisch, und es war unmenschlich, und das soll man nicht vergessen. +> Ich kann nicht die Greuel der NS-Verfolgung vergessen.

Aber man muss an andere Zeiten denken, man muss sehen, dass es vielleicht doch irgendwie anders sein kann. ${ }^{14}$

(2) Karl Sarközi: Keiner wusste am Anfang, wo wir hinkommen. Aber dann in Auschwitz haben wir es gesehen, was da los ist.

$+>$ Wir haben den Horror des KZ-Alltags gesehen.

$<\ldots>$ Wer das nicht mitgemacht hat, kann sich das nicht vorstellen. Und es gibt immer noch Millionen, die glauben alles nicht. Es gibt schon welche, die es glauben. Aber gerade hier, bei uns im Ort, wenn man es fünfzig erzählt... die glauben es nicht, die sagen, das ist nicht wahr. ${ }^{15}$

14 Interview mit Jehudith Hübner (2002): 2:38, https://www.weitererzaehlen.at/interviews/ jehudith-huebner-1 (Zugriff am 1.07.2020).

15 Interview mit Karl Sarközi (2006): 17:54 und 21:38, https://www.weitererzaehlen.at/interviews/karl-sarkoezi (Zugriff am 1.07.2020). 
(3) Interviewerin: Wann sind Sie zum ersten Mal wieder nach Österreich gefahren?

Esther Schuldmann: Lange her. Und sehr überraschend. Ich habe das überhaupt nie in Betracht gezogen. Jahrelang. Jahrzehntelang. Dann bin ich doch gefahren. Und unglücklich, denn ich kann nicht verzeihen, ich kann nichts verges$\underline{\text { sen. }}+$ > Ich kann nicht das mir zugetane Unrecht vergeben.

Interviewerin: Verständlich, ja. ${ }^{16}$

„Die Funktion des Tabus ist die des Schutzes von tabuisierten Inhalten“, ${ }^{17}$ folglich kann man vermuten, dass die SprecherInnen in den oben angeführten Beispielen der Andeutungen bewusst oder unbewusst explizite Verbalisierung von ihren traumatischen Erlebnissen meiden, um sich selbst zu schonen. Das bestätigen auch Erkenntnisse der psychotherapeutischen Forschungen: „Während nämlich so heikle Themen wie Sexualität, Mann-Frau-Probleme, Mutter/Töchter/ Söhne-Konflikte bereits feste Bestandteile jeder Therapeutenausbildung sind, ist das Tabu NS-Zeit auch hier ein „,blinder Fleck“ geblieben“ ${ }^{18}$ Dieses Tabu scheint bei den NS-Verfolgten trotz aller „Aufarbeitung der Geschichte“ immer noch gültig zu sein, denn ,,indem etwas tabuisiert wird, wird es auf eine spezifische Art und Weise fixiert und im Unbewußten konserviert" ${ }^{\text {"19 }}$. Nicht einmal die eigenen Kinder haben die Verfolgten an diesem Stück ihrer Lebensgeschichte teilhaben lassen:

(4) Interviewer: Haben Sie nach der NS-Zeit in der Familie über die Zeit geredet?

Erika Kosnar: Es ist eigentlich nie darüber geredet worden. $+>$ Ich habe niemandem von meinen Leiden erzählt. ${ }^{20}$

(5) Interviewerin: Wenn Sie sagen, ,wir haben furchtbar gelitten “- also wir kennen das zum Beispiel, dass man davon träumt. Haben Sie so was gehabt?

Chana Rubinstein: Und geschrien, ja, ja. Wie sagt man, solche Angstzustände. Weil das ist, die Erinnerung ist gekommen. Und wir wollten, die Kinder sollen frei sein. Und haben Gott sei Dank ein Land. Und sie waren frei. Wir wollten sie nicht belasten mit unseren Problemen. < ... Ich bin in einer Organisation, die heißt Amcha. Die ist im ganzen Land. Zum Beispiel, es gibt Veranstaltungen, es gibt Kurse. Und, ich bin in einer Gruppe mit sechs Leuten, mit einem Sozialarbeiter. Jeder hat was anderes mitgemacht. Und man erzählt Sachen, die man zu

16 Interview mit Esther Schuldmann (2006): 3:46, https://www.weitererzaehlen.at/interviews/ esther-schuldmann (Zugriff am 1.07.2020).

17 Rudas 1994: 17.

18 Hauer 1987: 30-31.

19 Erdheim 1993: 11.

20 Interview mit Erika Kosnar (2015a): 1:44:27, https://www.weitererzaehlen.at/interviews/ erika-kosnar (Zugriff am 1.07.2020). 
Hause nie erzählt hat. Nicht den Kindern, nicht der Frau. ${ }^{21}+>$ Jeder hat seine eigene traumatische Erfahrung der NS-Verfolgung, die er seinen Verwandten verschwiegen hat.

Für die Entscheidung, über diese Zeit zu schweigen, spricht auch die Erfahrung der Konfrontation mit GesprächspartnerInnen mit dem anderen Hintergrund: „Sprach man mit jemandem und erwähnte dabei Auschwitz, dann konnte man nahezu regelmäßig die gleiche Reaktion beobachten: Mitleidvoll wurde man angeblickt und der Gesprächspartner wich dem Thema aus, so, als ob er uns schonen wollte. Ich hatte die Empfindung, daß dieses Mitleid geheuchelt und eher die eigene Schonung beabsichtigt war". ${ }^{22}$ Das Verschweigen und Verdrängen in der Nachkriegszeit haben zwar auch die TäterInnen betrieben, doch aus anderen Gründen. Außerdem bedarf es „keiner Erklärung, daß jemand, der aus nationalsozialistischen Konzentrationslagern in das befreite Österreich zurückgekommen ist, dem, was man immer wieder als „Vergangenheitsbewältigung“ umschreibt, anders gegenübersteht als einer, der eine andere Vergangenheit hatte; der das „univers concentrationaire" - wie es David Russet genannt hat - nicht hat kennenlernen müssen". ${ }^{23}$

Durch die Euphemisierungsstrategie werden im Rahmen des Tabu-Clusters „traumatische Erfahrungen der NS-Verfolgung“ die verbalen Tabus aufgefangen, die für die deutschsprachige Sprachkultur generell gelten, es geht vor allem um solche tabuisierten Sachverhalte wie Gewalt, Tod, Mord, Vergewaltigung, Euthanasie, Sexualität, Zwangsprostitution, körperliche Funktionen etc., z.B.:

(6) Paul Grünberg: Es sind dann die Russen gekommen. Sie sind auch mit den Frauen nicht so zärtlich umgegangen. $+>$ Die Russen haben sie vergewaltigt.

Wir waren das Schutzschild für das Mädchen. Durch uns ist sie unberührt gewesen. ${ }^{24}$

(7) (Man redet über die Zwangsprostitution in Mauthausen.)

Interviewerin: Erzählen Sie mir, was die gemacht haben, mit den Frauen. $<$...>

+> Erzählen Sie über das KZ-Bordell.

Michael Horvath: Ja, ja, mei.

Interviewerin: Bitte.

Michael Horvath: Schande zum Erzählen. $<\ldots>$ Krepieren soll ich, aber Schande zum Erzählen, wirklich wahr.

${ }^{21}$ Interview mit Chana Rubinstein (2009): 2:26:30, https://www.weitererzaehlen.at/interviews/chana-rubinstein (Zugriff am 1.07.2020).

${ }^{22}$ Langbein 1987: 9.

23 Ebd., S. 8.

24 Interview mit Paul Grünberg (2014): 1:19:30, https://www.weitererzaehlen.at/interviews/ paul-gruenberg (Zugriff am 1.07.2020). 
Interviewerin: Herr Horvath, haben Ihnen die Frauen leidgetan, oder haben Sie sich gedacht, das ist wohl eine Frechheit, was die da machen? +> Haben Sie die Prostituierten verachtet?

Michael Horvath: Das kann ich nicht sagen, ich weiß es nicht, mit dem bin ich nicht in Verbindung gekommen, ich nicht. Ich habe sie nur gesehen und ich habe gewusst, was die sind und was sie mit den Kapo hatten. +> Sie waren Prostituierte und mussten mit den Kapo schlafen.

Aber geredet habe ich nicht mit ihnen, gar nicht. ${ }^{25}$

In dem oben angeführten Dialog muss auch die Interviewerin ihre Fragen durch Andeutungen umformulieren, weil ihr Interviewpartner sich weigerte, Sachen bei ihrem Namen zu nennen. Fokussiert werden die salienten Argumente der expliziten Proposition (was).

(8) (Man redet über die NS-Kindereuthanasieanstalt „Am Spiegelgrund“)

Alois Kaufmann: In der Schule war ein gewisser Plattner, das war ein Südtiroler, er war angeblich blöd, gestottert hat er ein bisschen, ein Kind. Der hat mir immer wieder erzählt, er war längere Zeit im 15er-Pavillon. Sagt er ,Weißt, bei uns stinkt es so. Und jeden zweiten, dritten Tag kommt ein Kind weg auf Erholung. Kommt nimmer her “, sag ich „Und du? “, „Na ja, vielleicht fahr ich auch einmal auf Erholung". Und so war es auch. Nach einer gewissen Zeit ist mein Freund, Schulkamerad Plattner nicht mehr gekommen und so war mir klar. $+>$ Mein Freund ist ermordet worden. ${ }^{26}$

(9) Alois Kaufmann: Groß, also die große Sache, hast du bis 60 zählen müssen, da ist einer vor der Klotüre gestanden und hat erzählt, ein Bursch, der neue Art Kapo-Stelle übernommen hat, Kinder-Kapo damals schon, und beim Lulu hast müssen bei 30 fertig sein. Wenn das nicht einer zusammenbracht hat, ist so wie bei den Texas-Türln so zum Auf- und Zumachen, ist er verhaut worden usw. ${ }^{27}$

\subsection{Tabu des expliziten Ausdrucks negativer Emotionen: Strategie des Gesichtswahrens}

Der zweite Tabu-Cluster erweckt besonderes Interesse, weil die Andeutungen hier die Strategie des Gesichtswahrens realisieren. In Interviews werden die Verfolgten auf ihr Verhältnis zu Österreich nach 1945 angesprochen. Hier lassen sich grob zwei Lager unterscheiden: diejenigen, die ihre negativen Gefühle den ÖsterreicherInnen gegenüber nicht verbergen, und wiederum diejenigen, die dem Interviewer/

25 Interview mit Michael Horvath (2003): 4:08 und 4:23:08, https://www.weitererzaehlen.at/ interviews/michael-horvath (Zugriff am 1.07.2020).

${ }^{26}$ Interview mit Alois Kaufmann (2015): 17:27, https://www.ueber-leben.at/home/alois-kaufmann (Zugriff am 1.07.2020).

27 Ebd., 05:43. 
der Interviewerin gegenüber über ihre negativen Gefühle „durch die Blume“ sprechen (der mögliche Grund für diese rücksichtsvolle Wortwahl wäre die Berücksichtigung der Tatsache, dass sie zwar ÖsterreicherInnen, und somit VertreterInnen des TäterInnenlandes, vor sich haben, die aber zu der zweiten, wenn nicht schon zu der dritten Generation gehören und womöglich durch die Täterschaft ihrer Vorfahren selbst traumatisiert sind). Somit bewahren sie das eigene Gesicht sowie das Gesicht des österreichischen Interviewers/der Interviewerin, z.B.:

(10) Interviewer: Haben Sie nach 1945 wieder mal gedacht nach Österreich zurückzukehren?

Jehudith Hübner: (schüttelt den Kopf) Nein. Niemals. Niemals. $<\ldots>\underline{\text { Ich war }}$ keine begeisterte Österreicherin nach dem, was ich dort erlebt habe. $<\ldots>+>$ Ich habe Österreich gehasst.

Interviewer: Hat sich Ihre Einstellung zu Österreich von 1945 bis heute geändert?

Jehudith Hübner: Österreich ist ein theoretischer Begriff. Ich rede über Menschen. $<\ldots>$ Es gab einen Haider, es gab einen Hitler. Es gab verschiedene Leute. die irgendwie nicht in Ordnung waren den Juden gegenüber. $+>$ Es gab viele Nazis.

Damit muss man leben.

Interviewer: Sprechen Sie im Alltag häufig Deutsch?

Jehudith Hübner: Nein (lächelt und schüttelt den Kopf). Nein. Nur wenn ich eingeladen werde zu Vorträgen. Freiwillig (lächelt) nein. $+>$ Die deutsche Sprache assoziiere ich mit meiner Verfolgung. Ich hasse sie.

Pause

Interviewer: Sie sagen, freiwillig nein...

Jehudith Hübner: Ich habe Freunde, die denselben Hintergrund haben wie ich, ja? Aber wir sprechen Hebräisch (lächelt) ${ }^{28}$. $+>$ Deutsch bleibt für uns die Sprache der TäterInnen.

In dem oben angeführten Dialog werden auch saliente non-verbale Indikatoren fokussiert - die Interviewte lächelt und signalisiert damit das Vorhandensein einer impliziten Botschaft in ihrer Aussage.

(11) Leo Luster: Meine Einstellung gegenüber Österreich hat sich verändert. Ich hab früher Österreich... (sucht nach dem passenden Wort) $+>$ Ich habe früher Österreich gehasst.

Ich wollte von Österreich nichts wissen, ja.

Interviewer: Verstehe ${ }^{29}$.

28 Interview mit Jehudith Hübner (2002): 2:29:46, https://www.weitererzaehlen.at/interviews/ jehudith-huebner-1 (Zugriff am 1.07.2020).

${ }^{29}$ Interview mit Leo Luster (2011): 2:17, https://www.weitererzaehlen.at/interviews/leo-luster-pfeifenberger (Zugriff am 1.07.2020). 
(12) Erika Kosnar: Ich habe es meinem Sohn erst nach der Matura gesagt, denn ich wollte nicht, dass er sich in der Schule verplaudert, und es hat zu der Zeit noch immer ewig Gestrige gegeben. $+>$ Es hat zu der Zeit noch immer Nazis gegeben.

Gibt's ja wiederum, dass er für etwas büßen muss, wofür er nichts kann ${ }^{30}$.

Während der Referenzpunkt der Andeutungen im ersten Tabu-Cluster in der Vergangenheit liegt, beziehen sich die Andeutungen im zweiten Tabu-Cluster auf die Gegenwart und eventuell auch auf die Zukunft, denn die SprecherInnen machen ihre Andeutungen mit Berücksichtigung der Tatsache, wer ihr aktueller Gesprächspartner ist (VertreterIn des TäterInnen-Landes, der/die aber zu der zweiten oder dritten Generation gehört), wenn sie ihre Aussage machen, und antizipieren die mögliche Wirkung ihrer Worte in der Zukunft.

\section{Fazit}

Die linguistische Untersuchung der Zeitzeuginnen-Interviews mit den NS-Verfolgten, die einen Bezug zu Österreich haben, hat zwei umfangreiche Tabu-Cluster aufgedeckt. Der erste Tabu-Cluster betrifft die traumatische Erfahrung der Verfolgung, die durch die kommunikative Strategie des Verschweigens und die der Euphemisierung bewältigt wird. Es geht hier vor allem um retrospektive Andeutungen. Der zweite Tabu-Cluster betrifft den expliziten Ausdruck von negativen Emotionen und wird durch die kommunikative Strategie des Gesichtswahrens aufgefangen. Es handelt sich dabei um introspektive und prospektive Andeutungen. $\mathrm{Zu}$ den Perspektiven der Forschung gehört eine vertiefte Untersuchung der genderbasierten Spezifik der Tabubewältigung.

\section{Literatur}

\section{Quellen}

Haslehner, Elfriede (2001): heldentaten. Lyrikflugblatt PODIUM.

Kaiser, Konstantin (2017): Nathan Fischmann. Lyrikflugblatt PODIUM.

Interview mit Paul Grünberg (2014:) https://www.weitererzaehlen.at/interviews/paul-gruenberg (Zugriff am 1.07.2020).

Interview mit Michael Horvath (2003): https://www.weitererzaehlen.at/interviews/michael-horvath (Zugriff am 1.07.2020).

30 Interview mit Erika Kosnar (2015b): 20:35, https://www.ueber-leben.at/home/erika-kosnar (Zugriff am 1.07.2020). 
Interview mit Jehudith Hübner (2002): https://www.weitererzaehlen.at/interviews/jehudith-huebner-1 (Zugriff am 1.07.2020).

Interview mit Alois Kaufmann (2015): https://www.ueber-leben.at/home/alois-kaufmann (Zugriff am 1.07.2020).

Interview mit Erika Kosnar (2015a): https://www.weitererzaehlen.at/interviews/erika-kosnar (Zugriff am 1.07.2020).

Interview mit Erika Kosnar (2015b): https://www.ueber-leben.at/home/erika-kosnar (Zugriff am 1.07.2020).

Interview mit Leo Luster (2011): https://www.weitererzaehlen.at/interviews/leo-luster-pfeifenberger (Zugriff am 1.07.2020).

Interview mit Chana Rubinstein (2009): https://www.weitererzaehlen.at/interviews/chana-rubinstein (Zugriff am 1.07.2020).

Interview mit Karl Sarközi (2006): https://www.weitererzaehlen.at/interviews/karl-sarkoezi (Zugriff am 1.07.2020).

Interview mit Esther Schuldmann (2006): https://www.weitererzaehlen.at/interviews/esther-schuldmann (Zugriff am 1.07.2020).

Interview mit Herbert Traube (2019): https://www.weitererzaehlen.at/interviews/herbert-traube, (Zugriff am 1.07.2020).

\section{Sekundärliteratur}

Bettelheim, Peter / Streibel, Robert (Hrsg.) (1994): Tabu und Geschichte. Zur Kultur des kollektiven Erinnerns. Wien: Picus.

Erdheim, Mario (1993): „I hab manchmal furchtbare Träume... Man vergißts Gott sei Dank immer glei... (Herr Karl)“. In: Ziegler, Meinrad / Kannonier-Finster, Waltraud (Hrsg.): Österreichisches Gedächtnis. Über Erinnern und Vergessen der NS-Vergangenheit. Wien/Köln/Weimar: Böhlau. S. 9-20.

Grice, Herbert Paul (1993): Logik und Konversation. In: Meggle, Georg (Hrsg.): Handlung, Kommunikation, Bedeutung. Frankfurt am Main: Suhrkamp. S. 243-265.

Hauer, Nadine (1987): NS-Trauma und kein Ende. In: Pelinka, Anton / Weinzierl, Erika (Hrsg.): Das große Tabu. Österreichs Umgang mit seiner Vergangenheit. Innsbruck/Wien: Edition S. S. $28-41$.

Langacker, Ronald (1990): Concept, Image and Symbol. The Cognitive Basis of Grammar. Berlin: de Gruyter.

Langacker, Ronald (2001): Discourse in Cognitive Grammar. In: Cognitive Linguistics 12-2, S. 143-148.

Langbein, Herrmann (1987): Darf man vergessen? In: Pelinka, Anton / Weinzierl, Erika (Hrsg.): Das große Tabu. Österreichs Umgang mit seiner Vergangenheit. Innsbruck/Wien: Edition S. S. $8-16$.

Liedtke, Frank (Hrsg.) (1995): Implikaturen. Grammatische und pragmatische Analysen. Tübingen: Niemeyer.

Meier, Christian (2010): Das Gebot zu vergessen und die Unabweisbarkeit des Erinnerns. Vom öffentlichen Umgang mit schlimmer Vergangenheit. München: Siedler.

Pelinka, Anton (1994): Tabus in der Politik. In: Bettelmann, Peter / Streibel, Robert (Hrsg.): Tabu und Geschichte. Zur Kultur des kollektiven Erinnerns. Wien: Picus. S. 21-28.

Pelinka, Anton / Weinzierl, Erika (Hrsg.) (1987): Das große Tabu. Österreichs Umgang mit seiner Vergangenheit. Innsbruck/Wien: Edition S.

Rolf, Eckard (2013): Inferentielle Pragmatik. Zur Theorie der Sprecher-Bedeutung. Berlin: Erich Schmidt Verlag. 
Rudas, Stephan (1994): Stichworte zur Sozialpsychologie der Tabus. In: Bettelheim, Peter / Streibel, Robert (Hrsg.): Tabu und Geschichte. Zur Kultur des kollektiven Erinnerns. Wien: Picus. S. $17-20$.

Ziegler, Meinrad / Kannonier-Finster, Waltraud (1993): Österreichisches Gedächtnis. Über Erinnern und Vergessen der NS-Vergangenheit. Wien/Köln/Weimar: Böhlau.

Zimmermann, Klaus / Müller, Peter (1977): Indirekte und implizite Sprechakte. In: Deutsche Sprache 5, S. 238-254. 